\title{
Upregulation of INS-IGF2 read-through expression and identification of a novel INS-IGF2 splice variant in insulinomas
}

\author{
LENE E. JOHANNESSEN ${ }^{1,2}$, IOANNIS PANAGOPOULOS ${ }^{1,2}$, SVEN-PETTER HAUGVIK $^{3,4}$, \\ IVAR PRYDZ GLADHAUG ${ }^{3,4}$, SVERRE HEIM ${ }^{1,2,4}$ and FRANCESCA MICCI ${ }^{1,2}$
}

\author{
${ }^{1}$ Section for Cancer Cytogenetics, Institute for Cancer Genetics and Informatics, The Norwegian Radium Hospital, \\ Oslo University Hospital, 0310 Oslo; ${ }^{2}$ Centre for Cancer Biomedicine, University of Oslo, 0379 Oslo; \\ ${ }^{3}$ Department of Hepato-Pancreato-Biliary Surgery, Rikshospitalet, Oslo University Hospital, 0372 Oslo; \\ ${ }^{4}$ Institute of Clinical Medicine, University of Oslo, 0450 Oslo, Norway
}

Received April 4, 2016; Accepted June 30, 2016

DOI: $10.3892 /$ or.2016.5132

\begin{abstract}
Fusion transcripts arising from the combination of exons residing on neighboring genes on the same chromosome may give rise to chimeric or novel proteins. Such read-through transcripts have been detected in different cancers where they may be of pathogenetic interest. In this study, we describe for the first time the expression of a read-through transcript in insulinomas, a functioning neuroendocrine pancreatic neoplasm. The read-through transcript INS-IGF2, composed of exons from the two genes proinsulin precursor (INS) and insulin-like growth factor 2 (IGF2), both mapping to chromosomal subband $11 \mathrm{p} 15.5$, was highly expressed in the two insulinomas analyzed. More precisely, version 2 of the INS-IGF2 transcript was expressed, indicating possible expression of the chimeric INS-IGF2 protein. We further identified a novel splice variant of the INS-IGF2 read-through transcript in one of the insulinomas, composed of exon 1 of $I N S 3$ and exons of $I G F 2$. In the same tumor, we found high expression of INS3 and the presence of the A allele at SNP rs689. SNP rs689 has been previously described to regulate splicing of the INS transcript, indicating that this regulatory mechanism also affects splicing of INS-IGF2. The identification of the INS-IGF2 read-through transcript specifically in tumor tissue but not in normal pancreatic tissue suggests that high expression of INS-IGF2 could be neoplasia-specific. These results may have potential clinical applications given that the read-through transcript could be used as a biomarker in insulinoma patients.
\end{abstract}

Correspondence to: Dr Francesca Micci, Section for Cancer Cytogenetics, Institute for Cancer Genetics and Informatics, The Norwegian Radium Hospital, Oslo University Hospital, Ullernschausseen 70,0310 Oslo, Norway

E-mail: francesca.micci@labmed.uio.no

Key words: INS-IGF2, insulinomas, transcriptome sequencing, proinsulin precursor, read-through transcript

\section{Introduction}

Insulinomas are neuroendocrine neoplasms derived from the $\beta$-cells of the pancreas. They belong to the group of pancreatic neuroendocrine tumors (PNETs). Most insulinomas are benign with only $10 \%$ showing malignant features $(1,2)$; in the latter situation, the liver and regional nymph nodes are the main sites of metastases (3-5). Despite the general pancreatic origin of insulinomas, there are reports of some cases originating from ectopic pancreatic tissue $(6,7)$. Although rare, insulinomas represent the most common functioning pancreatic neuroendocrine neoplasm with an incidence of 1-4 per million per year (1). The only curative treatment is complete surgical resection (8).

PNETs occur sporadically or are inherited as part of the multiple endocrine neoplasia type 1 (MEN1) syndrome. Individuals with MEN1 syndrome have constitutional mutations in the MEN1 gene which render them genetically predisposed to endocrine tumors. Although insulinomas account for $10-30 \%$ of pancreatic tumors in patients with MEN1, only few patients with insulinomas harbor MENI mutations (4-6\%) (1,9). Little is known about the other genetic alterations in insulinomas and it has been shown that the most common oncogenes are uninvolved in PNETs (10). Comparative genomic hybridization (CGH) studies have demonstrated genomic imbalances in insulinomas (9-17). High frequencies of gains at 5p, 5q, 7p, 7q, 9p, 9q, 12q, 14q, 17p, 17q, and $20 \mathrm{q}$ were detected whereas frequent losses were scored at 1p, 1q, 2q, 3q, 9p, 6p, 6q, 10q, 11p, 11q, and 22q $(10,13)$. Chromosomal band 9q34 was found gained in 50\% of insulinomas $(11,13)$. Furthermore, array-CGH and FISH analyses showed that gain of $9 \mathrm{q} 32$ and loss of 22q13.1-q13.31 are early and independent genetic events in insulinomas while losses of 11q23.3-q24.3 and 22q13.31-q13.32 are associated with tumor development (12).

Gene fusions are common and pathogenetically essential in many types of neoplasia. The chimeric fusions and resulting abnormal protein products may not only be important in tumorigenesis, but they also constitute ideal diagnostic features and may be therapeutic targets (18). Since the major genetic alterations in sporadic insulinomas are still unknown, 
we decided to perform RNA sequencing on two such tumors looking for possible fusion transcripts.

\section{Materials and methods}

Ethics statement. The study was approved by the regional ethics committee (Regional Komité for Medisinsk Forskningsetikk Sør-Øst, Norge, http://helseforskning.etikkom.no; project number 2011/1945), and written informed consent was obtained from the patients.

Case history. Case 42: A 37-year-old female showing unexplained fatigue, rapid heartbeat, trouble thinking clearly, and repetitive episodes where low blood sugar values were measured was referred to our hospital for further examination. Biochemical evidence of insulinoma was found. Cross-sectional imaging showed a small solid lesion in the pancreatic tail but no sign of metastatic disease. A laparoscopic distal spleen-preserving pancreatectomy was performed. Histology showed a $13 \mathrm{~mm}$ insulinoma with a Ki-67 of $6 \%$ and ENETS TNM stage pT1N0 (data not shown). There was no sign of recurrence 25 months after surgery (19).

Case 59: A 30-year-old male with a known history of acute lympoblastic leukemia (ALL) in remission was admitted to our hospital with left-sided abdominal and lower back pain. Shortly afterwards he had an episode of unconsciousness and severe hypoglycemia was measured. Cross-sectional imaging showed a large left-sided retroperioneal tumor with infiltration of the adrenal gland and kidney. Because a malignant adrenal tumor was suspected, the patient underwent concomitant left nephrectomy and adrenalectomy. Intraoperatively, suspicious liver lesions were detected which were biopsied. Histology showed a malignant insulinoma with a Ki-67 of 38\%. Postoperative octreotide scintigraphy and FDG-PET/CT showed pathologic uptake in the pancreas and liver as well as in the thoracic lymph nodes. Medical treatment was started (temozolomide, everolimus, bevacizumab). Due to continuous hypoglycemic symptoms the patient was referred for debulking surgery. A laparoscopic distal spleen-preserving pancreatectomy and a simultaneous laparoscopic resection of two metastases in the right liver lobe were performed. Histology showed a $15-\mathrm{mm}$ insulinoma in the pancreatic tail with a $\mathrm{Ki}-67$ of $9 \%$ and ENETS TNM stage pT1N1M1 (data not shown). The patient has stable disease after ongoing medical treatment 27 months after the first surgery (19).

Case 45: The control sample was harvested from normal pancreatic tissue from a 40-year-old male who underwent laparoscopic distal pancreatectomy with splenectomy for a nonfunctioning pancreatic neuroendocrine neoplasm. Histology of the pancreatic lesion showed neuroendocrine morphology with a Ki-67 of $2 \%$ and ENETS TNM stage pT1N0 (data not shown). The control sample was harvested from the same surgical specimen separated from the actual neuroendocrine lesion. The patient has no sign of recurrence of the disease 40 months after surgery.

High-throughput paired-end RNA-sequencing. Tumor tissue adjacent to that used for histologic examination was frozen and stored at $-80^{\circ} \mathrm{C}$. Total RNA was extracted from the insulinomas (cases 42 and 59) and one control sample (case 45) using miRNeasy Mini kit according to the manufacturer's instructions (Qiagen Nordic, Oslo, Norway). Tumor tissue was disrupted and homogenized in QIAzol Lysis Reagent using a $5 \mathrm{~mm}$ stainless steel bead and TissueLyser II (both from Qiagen). Subsequently, total RNA was purified using QIAcube (Qiagen). The RNA quality was evaluated using the Experion automated electrophoresis system (Bio-Rad Laboratories, Hercules, CA, USA). Three micrograms of total RNA for cases 42 and 59 were sent for high-throughput paired-end RNA-sequencing at the Norwegian Sequencing Centre, Ullevål Hospital (http://www.sequencing.uio.no/). The RNA was sequenced using an Illumina HiSeq 2000 instrument and the Illumina software pipeline was used to process image data into raw sequencing data. The TruSeq Stranded mRNA sample preparation protocol was used (http://support.illumina. com/downloads/truseq_stranded_mrna_sample_preparation guide_15031047.ilmn) giving reads of a length of 100 base pairs. The Illumina software pipeline was used to process image data into raw sequencing data and only sequence reads marked as 'passed filtering' were used in the downstream data analysis. A total of 103 and 98 millions reads, respectively, were obtained. The FastQC software was used for quality control of the raw sequence data (http://www.bioinformatics.babraham. ac.uk/projects/fastqc/). We used the fusion discovery software FusionMap (release date 16-04-2012) and the pre-built Human B37 and RefGene from the FusionMap website (http://www. omicsoft.com/fusionmap/) (20).

$R T$-PCR. RNA was purified for cases 42 and 59 as well as for the normal tissue used as the control (case 45). The purification was as described in the 'High-throughput paired-end RNA-sequencing' method-section except that an optional 'on column' DNase-treatment step was included as recommended by the manufacturer (Qiagen). Five micrograms of total RNA were reverse-transcribed in a $100 \mu \mathrm{l}$ reaction volume using iScript Advanced cDNA Synthesis Kit for RT-qPCR according to the manufacturer's instructions (Bio-Rad Laboratories). For case $45,1.33 \mu \mathrm{g}$ of total RNA were reverse-transcribed in a $40 \mu 1$ reaction volume. RNA was diluted in RNase-free water to a concentration equivalent to $15 \mathrm{ng} / \mu 1$ DNase treated RNA. For PCR reactions requiring higher start-amounts of template, $2 \mu \mathrm{g}$ of RNA from the 'RNA-sequencing' method-section was reverse-transcribed in a $20 \mu \mathrm{l}$ reaction volume before being diluted in RNase-free water to a concentration equivalent to $50 \mathrm{ng} / \mu 1 \mathrm{RNA}$.

The $25 \mu 1$ PCR volume contained $12.5 \mu 1$ of Premix Ex Taq Hot Start (Takara Bio Inc., Otsu, Japan), 0.25-2 $\mu 1$ of cDNA, and $0.4 \mu \mathrm{M}$ of each of the forward and reverse primers. The primers used for PCR amplification and sequencing are listed in Table I (for primer localization, see Fig. 1). For primer combinations, cDNA concentration and volume of cDNA used, see figure legends and Table II. The PCR conditions for all amplifications, except for primer combinations $4+6$, $4+7$, and $3+5$, were: an initial denaturation at $94^{\circ} \mathrm{C}$ for $30 \mathrm{sec}$ followed by 35 cycles of $7 \mathrm{sec}$ at $98^{\circ} \mathrm{C}$ and $2 \mathrm{~min}$ at $68^{\circ} \mathrm{C}$, and a final extension for $5 \mathrm{~min}$ at $68^{\circ} \mathrm{C}$. For the amplifications using the primer sets $4+6,4+7$ or $3+5$, the PCR conditions were: an initial denaturation at $94^{\circ} \mathrm{C}$ for 1 min followed by 35 cycles of $10 \mathrm{sec}$ at $98^{\circ} \mathrm{C}, 1 \mathrm{~min}$ at $66^{\circ} \mathrm{C}$ and $2 \mathrm{~min}$ at $72^{\circ} \mathrm{C}$, followed by a final extension for $5 \mathrm{~min}$ at $72^{\circ} \mathrm{C}$. The PCRs were run 
Table I. Primers used for RT-PCR analysis.

\begin{tabular}{cllclll}
\hline & & \multicolumn{2}{c}{ Target } & \\
\cline { 3 - 5 } Primer & \multicolumn{1}{c}{ Name } & Transcript & Isoform & Accession no. & Exon & \multirow{2}{*}{ Sequence } \\
\hline \multirow{2}{*}{1} & INSIGF2-96F & $I N S$ & 1 & NM_000207.2 & Ex2 & CTGCTGGCCCTCTGGGGACCT \\
2 & INS1-388R & $I N S$ & 1 & NM_000207.2 & Ex3 & GCCTGCGGGCTGCGTCTAGT \\
3 & INS2-42F & $I N S$ & 2 & NM_001185097.1 & Ex1 & GGTCTGTTCCAAGGGCCTTTGCGT \\
4 & INSIGF2-55F & $I N S$ & 1 & NM_000207.2 & Ex2 & CTGCCATGGCCCTGTGGATGCG \\
5 & INSIGF2-378R & $I G F 2$ & 2 & NM_001007139.5 & Ex1 & GGTGCCCAAGGCTCTCTGCCG \\
6 & INSIGF2_1-1000R & $I G F 2$ & 2 & NM_001007139.5 & Ex4 & CGGAAGCACGGTCGGAGGGGT \\
7 & INSIGF2_2-752R & $I N S-I G F 2$ & 2 & NM_001042376.2 & Ex5 & CCCGGCTTCTATCTGGGATGGGCA \\
8 & IGF2-48F & $I G F 2$ & 2 & NM_001007139.5 & Ex1 & CCCCAGCGGCCTCAGCACTAC \\
9 & ABL-91F & $A B L$ & A & NM_005157.5 & Ex2 & CAGCGGCCAGTAGCATCTGACTTTG \\
10 & ABL-404R1 & $A B L$ & A & NM_005157.5 & Ex3 & CTCAGCAGATACTCAGCGGCATTGC \\
\hline
\end{tabular}

A

B

Chromosome 11p15.5:

$2,161,205$ bp
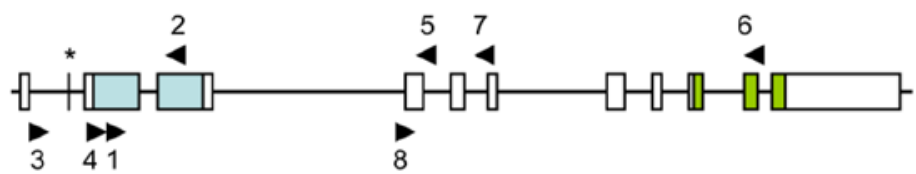

$2,129,112 b p$

Transcript Version

INS $\left\{\begin{array}{l}1 \\ 2 \\ 3 \\ 4\end{array}\right.$
IGF2 $\left\{\begin{array}{l}1 \\ 2 \\ 3 \\ 4 \\ 5\end{array}\right.$

INS-IGF2 $\left\{\begin{array}{l}1 \\ 2\end{array}\right.$
Accesion no

NM_000207.2

NM_001185097.1

NM_001185098.1

NM_001291897.1

NM_000612.5

NM_001007139.5

NM_001127598.2

NM_001291861.2

NM 001291862.2

NR_003512.3

NM_001042376.2
Schematic diagram
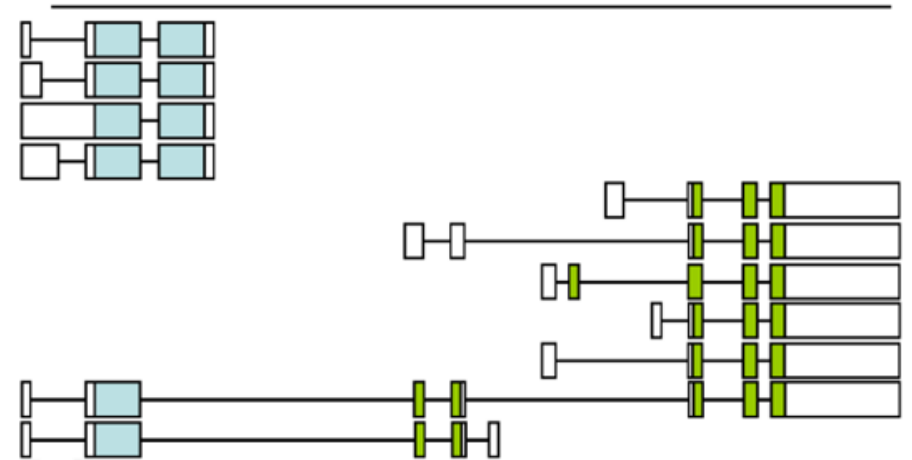

Figure 1. Schematic scale diagram of the INS/IGF2 domain on human chromosome 11. (A) Schematic organization of exons in the INS/IGF2 domain on human chromosome subband 11p15.5. Exons that are part of INS1-4, isoform 2 of IGF2, and the read-through INS-IGF2 are shown. Coding exons are colored. Approximate positions of the RT-PCR primers are indicated by arrowheads. The position of the SNP rs689 is indicated by an asterisk. (B) Schematic diagram of the human INS, IGF2, and INS-IGF2 transcripts. Coding exons are colored.

on a C-1000 Thermal cycler (Bio-Rad Laboratories). Four or $10 \mu \mathrm{l}$ of the PCR products were analyzed by electrophoresis through a 1.5 or $1 \%$ agarose gel stained with GelRed (Biotium, Inc., Hayward, CA, USA), and photographed. The PCR products were purified using the QIAquick PCR purification kit (Qiagen) or the QIAquick Gel extraction kit (Qiagen) and direct sequencing was performed using the light run sequencing service of GATC Biotech (http://www.gatc-biotech. com/en/sanger-services/lightrun-sequencing.html). The BLAST software (http://www.ncbi.nlm.nih.gov/BLAST/) was used for computer analysis of the sequence data.

Real-time PCR. DNase-treated RNA for cases 42, 59, and 45, purified as detailed in the 'RT-PCR' method section, was diluted in RNase-free water to a concentration equivalent to $30 \mathrm{ng} / \mu 1$ DNase-treated RNA. For quantification of the expression of the proinsulin precursor $(I N S)$, insulin-like growth factor 2 (IGF2), INS-IGF2, and $\beta$-actin $(A C T B)$ transcripts, four TaqMan-based real-time assays with FAM-MGB-labelled probes were performed, all supplied by Applied Biosystems (Thermo Fisher Scientific Inc., Waltham, MA, USA). Assay Hs04185271_g1 was used for expression of the INS-IGF2 read-through transcript. This assay is specific for the exon 2-3 boundary of INS-IGF2 and detects both isoforms of the INS-IGF2 read-through transcript (accession nos. NR_003512.3 and NM_001042376.2). Assay Hs02741908_m1 was used for the expression of INS. This assay is specific for the exon 2-3 boundary of INSI and detects all four isoforms (accession nos. NM_000207.2, NM_001185097.1, NM_001185098.1, and NM_001291897.1). Assay Hs04194920_s1 was used for detection of IGF2. This assay is specific for exon 1 of isoform 2 of IGF2 (accession no. NM_001007139.5), with a probe located at a region not present in the INS-IGF2 transcripts (249 bp in isoform 2 of $I G F 2$ is located within the probe). Isoform 2 is the isoform of $I G F 2$ that is part of the INS-IGF2 read-through transcript. The 
Table II. Primer combinations used for RT-PCR.

\begin{tabular}{llcc}
\hline $\begin{array}{l}\text { Primer } \\
\text { combination }^{\mathrm{a}}\end{array}$ & Transcript & Isoform & Size (bp) \\
\hline $1+2$ & INS & 1 & 312 \\
& & 2 & 312 \\
& & 4 & 312 \\
$3+2$ & INS & 2 & 312 \\
& & 3 & 392 \\
& & 4 & 545 \\
$4+5$ & INS-IGF2 & 1 & 346 \\
& & 2 & 344 \\
$4+6$ & $I N S-I G F 2$ & 1 & 966 \\
$4+7$ & $I N S-I G F 2$ & 2 & 721 \\
$3+5$ & $I N S-I G F 2$ & Novel & $383 / 536 / 417^{\mathrm{b}}$ \\
$8+5$ & $I G F 2$ & 2 & 363 \\
$9+10$ & $A B L$ & $\mathrm{~A}$ & 338 \\
\hline
\end{tabular}

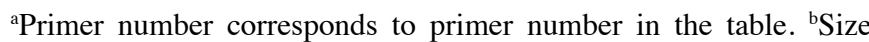
expected when INS-IGF2 is made by splicing of INS2 and IGF2, $I N S 3$ and $I G F 2$, and INS4 and IGF2 respectively.

assay Hs01060665_g1 is specific for the exon 2-3 boundary of ACTB (accession no. NM_001101.3) and was used as an endogenous control for relative gene expression quantification. To quantify the expression of the INS transcript with a deletion in exon 3, primers and a TaqMan probe were generated to span this region. The primers and probe used were: forward, 5'-TGC AGGCTGGAGAA-3'; reverse, 5'-CTGGTTCAAGGGCT TTA-3'; and probe, 5'-CGCCTCCTGCACCGA-3'. The probe was labelled with FAM-MGB. A 20X concentrated mixture of these generated primers and probe was made (INSdel-mix) containing $18 \mu \mathrm{M}$ of each primer and $5 \mu \mathrm{M}$ of the probe, which is identical to the concentrations used in the 20X TaqMan Gene Expression Mix (Applied Biosystems). Four replicates of each sample were used to ensure statistical representativity. The $20 \mu 1$ reaction volume contained 1X TaqMan Universal Mix, 1X TaqMan Gene Expression Mix or 1X INSdel mix, and $1 \mu \mathrm{l}$ cDNA (concentration equivalent to $30 \mathrm{ng} / \mu 1$ DNase-treated RNA). Real-time PCR was run on a CFX96 Touch ${ }^{\mathrm{TM}}$ Real-Time PCR Detection system (Bio-Rad Laboratories). The thermal cycling included an initial step at $50^{\circ} \mathrm{C}$ for $2 \mathrm{~min}$, followed by $10 \mathrm{~min}$ at $95^{\circ} \mathrm{C}$ and 40 cycles of $15 \mathrm{sec}$ at $95^{\circ} \mathrm{C}$ and $1 \mathrm{~min}$ at $60^{\circ} \mathrm{C}$. The data were analyzed using the Bio-Rad CFX Manager software (Bio-Rad Laboratories) and the Microsoft ${ }^{\circledR}$ Excel Software (Microsoft Corporation, Redmond, WA, USA). Expression of the different transcripts was normalized to $A C T B$ expression before calculation of the relative expression level using the comparative $\mathrm{Cq}$ method $(\Delta \Delta \mathrm{Cq})(21)$.

\section{Results}

High-throughput paired-end RNA-sequencing analysis. Using the FusionMap software with the fastq files obtained from the
Norwegian Sequencing Centre, Ullevål Hospital (http://www. sequencing.uio.no/), a list of $>1,000$ possible fusion genes was obtained for both tumors (1,018 and 1,159, respectively). Involvement of the INS and the IGF2 genes was noted in both. More specifically, involvement of the INS-IGF2 read-through as one of the fusion partners ranked as number two and three in case 42, with 98 and 66 reads, respectively (164 reads in total) (Table III). In all, a total of 472 reads showed the involvement of INS, IGF2 and/or the read-through INS-IGF2 as one of the fusion partners in case 42. Case 59 showed ten fusions involving $I N S, I G F 2$ and/or the read-through INS-IGF2 with different partners (Table III). As FusionMap detects INS-IGF2 as a fusion partner, this read-through will not be detected by the software as a fusion by itself.

Expression of different INS transcripts. RNA sequencing identified possible involvement of exons from the INS and $I G F 2$ genes in formation of fusion genes. The INS and $I G F 2$ loci are located sequentially on chromosome subband 11p15.5 (Fig. 1). Notably, these loci also encode the previously described INS-IGF2 read-through transcript (22), a fusion transcript containing exons of the INS locus 5 ' fused to exons of the IGF2 locus 3' (Fig. 1). The detection of transcripts by RNA sequencing that could potentially involve the INS-IGF2 read-through transcript caught our interest as read-through transcripts have not been described in insulinomas. We therefore decided to investigate in more detail whether the INS-IGF 2 transcript itself could be expressed in insulinomas.

In order to investigate the possible expression of INS-IGF2 in insulinomas, we performed PCR analysis of the two insulinomas together with a control sample from normal pancreatic $\beta$-cells (case 45) (Fig. 2; for primer combinations, Table II). INS-IGF2 read-through PCR-fragments could be detected in both insulinomas but not in the control sample (Fig. 2A). Sanger sequencing confirmed the identity of the PCR-fragments, clearly identifying expression of the read-through INS-IGF2 in insulinomas. Transcripts of the INS gene could be detected in all samples, although the expression level was clearly higher in both insulinomas compared to the control (Fig. 2A). A PCR-fragment with a lower molecular weight than the INS PCR-fragment (called INSdel) was detected in all samples upon PCR-detection of INS, showing the same expression pattern as INS with higher expression in the insulinomas (Fig. 2A). Sanger sequencing identified this INSdel transcript as a shorter version of the INS transcript, with a deletion of the first $125 \mathrm{bp}$ in exon 3 (Fig. 2B and C). This short INS-transcript was identical to one of the suggested alternative $I N S$-transcripts detected by transcriptome sequencing. The INSdel transcript has an out-of-frame deletion and lacks a STOP-codon, giving a novel non-coding $I N S$-transcript. IGF2 could also be detected in all samples with a clearly higher expression level in insulinomas than in the control sample (Fig. 2A). Two additional control samples from normal pancreatic $\beta$-cells showed the same expression pattern of INS-IGF2 and INS as the control sample used in this study (data not shown). Due to the limited material available, these two control samples were not included.

To quantify the relative expression of INS-IGF2 read-through transcript, real-time PCR was performed (Fig. 3). Expression of the different transcripts was normalized to $A C T B$ expression before the relative expression was calcu- 
Table III. Fusion transcripts involving INS, IGF2 and/or the read-through INS-IGF2 detected upon RNA-sequencing analysis.

\begin{tabular}{|c|c|c|c|c|c|c|}
\hline Case $^{a}$ & Rank & $5 '$ gene & $5^{\prime}$ gene junction & 3' gene & $3^{\prime}$ gene junction & Seed reads \\
\hline \multirow[t]{10}{*}{42} & 1 & SPTBN5 & $\operatorname{chr15:42164047}$ & INS & chr11:2181187 & 443 \\
\hline & 2 & $I N S-I G F 2, I G F 2$ & chr11:2170573 & $I N S$ & chr11:2181184 & 98 \\
\hline & 3 & $I N S-I G F 2, I G F 2$ & chr11:2170575 & INS & chr11:2181101 & 66 \\
\hline & 18 & $I N S-I G F 2, I N S$ & chr11:2182184 & $P S A P$ & chr10:73576794 & 13 \\
\hline & 25 & $I N S-I G F 2, I N S$ & $\operatorname{chr11:2182184}$ & $A U R K A I P 1$ & chr:11309510 & 11 \\
\hline & 30 & $I N S-I G F 2, I N S$ & chr11:2182063 & $F A M 159 B$ & chr5:63991473 & 9 \\
\hline & 35 & $C H G A$ & chr14:93401433 & INS & chr11:2181068 & 8 \\
\hline & 36 & $I N S-I G F 2, I N S$ & chr11:2182159 & $E D F 1$ & chr9:139757890 & 8 \\
\hline & 37 & $I N S-I G F 2, I N S$ & chr11:2182193 & $F T L$ & chr19:49469598 & 8 \\
\hline & 44 & $I N S-I G F 2, I N S$ & chr11:2182147 & $G A P D H$ & chr12:6646957 & 7 \\
\hline \multirow[t]{10}{*}{59} & 37 & SPTBN5 & chr15:42164047 & INS & chr11:2181187 & 10 \\
\hline & 716 & RERI & chr1:2336736 & $I N S-I G F 2, I N S$ & $\operatorname{chr11:2182217}$ & 2 \\
\hline & 717 & $J T B$ & chr1:153949707 & $I N S-I G F 2, I N S$ & chr11:2182028 & 2 \\
\hline & 724 & $I N S-I G F 2, I G F 2$ & chr11:2152930 & GPR123 & chr10:134944378 & 2 \\
\hline & 743 & INS & $\operatorname{chr11:2181214}$ & WBP11 & chr12:14940366 & 2 \\
\hline & 805 & $I N S-I G F 2, I N S$ & chr11:2182184 & PDIA3 & chr15:44038744 & 2 \\
\hline & 868 & COL1A1 & $\operatorname{chr17:48262336}$ & INS & $\operatorname{chr11:2181174~}$ & 2 \\
\hline & 869 & $I N S-I G F 2, I N S$ & $\operatorname{chr11:2182167}$ & $P N P O$ & $\operatorname{chr} 17: 46026276$ & 2 \\
\hline & 870 & $I N S-I G F 2, I N S$ & chr11:2182202 & VPS 25 & chr17:40925822 & 2 \\
\hline & 939 & $P R K D 2$ & chr19:47177673 & $I N S-I G F 2, I N S$ & chr11:2182075 & 2 \\
\hline
\end{tabular}

${ }^{\mathrm{a}}$ For case 42, 10 out of 472 reads are shown. For case 59, all 10 reads involving INS, IGF2 and/or the read-through INS-IGF2 are shown.

lated $\left(2^{-\Delta \Delta \mathrm{Cq}}\right)$ and set as 1 for case 42 . We could clearly detect INS-IGF2 in the two insulimonas (Cq values were $30.13 \pm 0.069$ and $33.12 \pm 0.063$ for cases 42 and 59 , respectively) but were unable to detect expression in the control sample. INS expression was high in the insulinomas (Cq values, 19.21 \pm 0.186 and $23.81 \pm 0.626$ for cases 42 and 59, respectively) compared to the control case (Cq value, $35.60 \pm 0.529)$, giving an increase in INS expression in the insulinomas of $\sim 2.5 \times 10^{5}$ and $4 \times 10^{3}$ fold for cases 42 and 59, respectively, when comparing the $2^{-\Delta \Delta \mathrm{Cq}}$ values. Expression of the short INS transcript with an out-of-frame deletion (INSdel) could only be detected in the two insulinomas (Cq values, 29.70 \pm 0.340 and $29.60 \pm 0.431$ for cases 42 and 59, respectively). Additionally, expression of $I G F 2$ version 2 was increased in the two insulinomas $(\mathrm{Cq}$ values, $32.08 \pm 0.568$ and $32.09 \pm 0.034$ for cases 42 and 59, respectively) compared to the control sample where IGF2 could only be detected in one out of the four replicates ( $\mathrm{Cq}$ value, 39.60). The expression levels of the four transcripts, INS-IGF2, INS, INSdel, and IGF2 version 2, were all clearly higher in one of the insulinomas (case 42; Fig. 3).

Molecular genetic confirmation of different INS-IGF2 transcripts. Two INS-IGF2 transcripts have been described (Fig. 1) (22). Transcript version 1 is a long transcript that is a candidate for nonsense mediated decay (NMD; accession no. NR_003512.3). Transcript version 2 is translated into a protein of 200 aa (accession no. NM_001042376.2). As translation of $I N S-I G F 2$ transcript into protein may be dependent upon the version of INS-IGF2 transcript present, we performed additional PCR-analyses with primers specific for either transcript version 1 or transcript version 2 of $I N S-I G F 2$. We were not able to detect the transcript version 1 of INS-IGF2 (data not shown). However, a PCR fragment for transcript version 2 was detected in the two insulinomas but not in the normal control (Fig. 4A). Sanger sequencing of the PCR-fragment confirmed that this fragment was the transcript version 2 of INS-IGF2 (Fig. 4B and C).

Alternative splicing in the 5'-untranslated region of INS gives rise to four INS transcripts coding for the same protein (http://www.ncbi.nlm.nih.gov/nucleotide/) (Fig. 1). The first $26 \mathrm{bp}$ of intron 1 in the INS1-transcript are present in exon 1 of the INS2-4 transcripts and has been postulated to increase the translational efficiency of the INS protein (23). In insulinomas, increased expression of the INS2-transcript has been reported (23). As INS and INS-IGF2 share the same transcriptional start site, we wanted to investigate whether the alternative splicing mechanisms described for INS, regulating a switch from INS1 to INS2, may also affect the INS-IGF2 read-through transcript. PCR-analysis of the 5'-untranslated region of INS-IGF2 and INS using a forward primer specific for the reported 5' 26 bp intron 1 sequence of INS1 (23), the forward primer only recognizing INS2-4, detected high expression of INS in the two insulinomas (Fig. 5A). In one of them (case 59), two bands were detected. Sanger sequencing identified the higher band in case 59 as INS3, the lower band had a readable sequence found in all four INS isoforms, identifying this band as INS2 and/or INS4. For INS-IGF2, a band with a higher molecular weight than expected was detected in case 
A

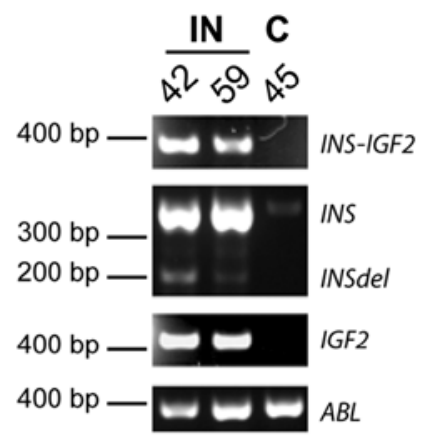

B
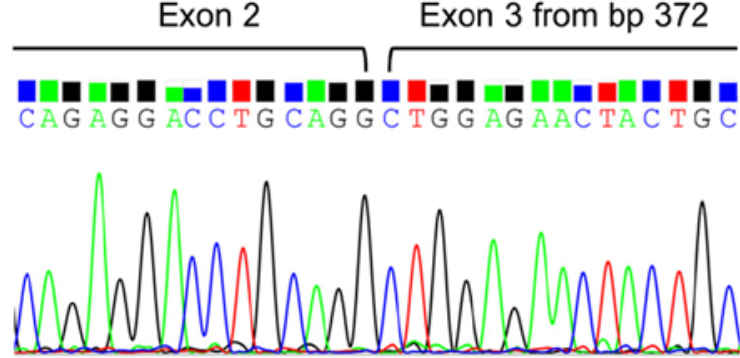

C

SEQUENCE

INS1

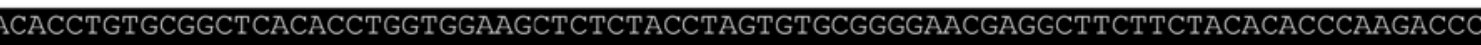

ACACCTGTGCGGCTCACACCTGGTGGAAGCTCTCTACCTAGTGTGCGGGGAACGAGGCTTCTTCTACACACCCAAGACCC

SEQUENCE 81 GCCGGGAGGCAGAGGACCTGCAG

INS1 223 GCCGGGAGGCAGAGGACCTGCAGC

SEQUENCE 105

INS1

303 CTGGAGGGGTCCCTGCAGAAGCGTGGCATTGTGGAACAATGCTGTACCAGCATCTGCTCCCTCTACCAGC

\section{SEQUENCE 116 CTGCAACTAGACGCAGCCCGCAGG \\ INS1 383 CTGCAACTAGACGCAGCCCGCAGG}

Figure 2. Expression of INS-IGF2 in insulinomas. Expression of the INS-IGF2 transcripts was analyzed in the two insulinomas (cases 42 and 59) and in one control sample (case 45). (A) INS-IGF2 was amplified from $1 \mu 1$ cDNA with a concentration equivalent to 15 ng/ $\mu 1$ DNase-treated RNA using primers 4 and 5, INS was amplified using primers 1 and 2, and IGF2 was amplified using primers 8 and 5. ABL (NM_005157.5) was amplified using primers 9 and 10 and used as an internal control for the PCR reaction. A PCR fragment with a lower molecular weight than the INS PCR-fragment (called INSdel) was detected in all samples upon PCR detection of INS. (B) Part of the sequence of the INSdel transcript amplified by RT-PCR in (A). Indicated are the exon-exon junction between exon 2 and the exon 3 lacking 125 bp 5'. (C) Sequence alignment of the INSdel sequence amplified in (A).

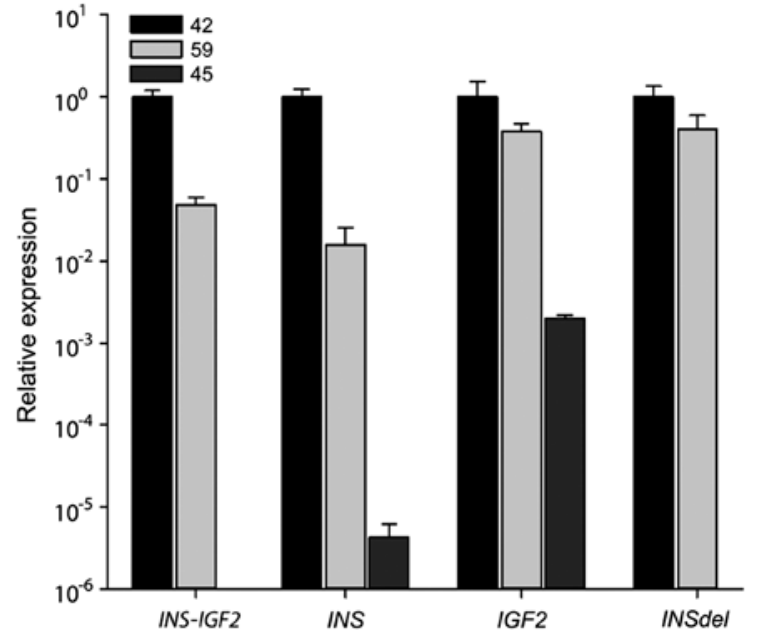

Figure 3. Real-time PCR quantification of INS-IGF2 expression in insulinomas. Quantification was performed as described in the 'Materials and methods' section. The data were analyzed using the Bio-Rad CFX Manager software (Bio-Rad Laboratories) and the Microsoft ${ }^{\circledR}$ Excel Software (Microsoft). Expression of the different transcripts was normalized to ACTB expression before calculation of the relative expression level using the comparative Cq method $(\Delta \Delta \mathrm{Cq})$. Case 42 was used as calibrator for calculation of $\Delta \Delta \mathrm{Cq}$ in order to give a relative expression of one $\left(2^{-\Delta \Delta \mathrm{Cq}}\right)$ for the different transcripts in case 42 . Mean relative expression of the four replicates is shown \pm the expression range.

59 (Fig. 5B). Sanger sequencing identified this fragment as a novel splice variant of INS-IGF2, containing exon 1 of INS3 spliced to exon 1 of IGF2 (Fig. 6A and B). Upon examina- tion of the novel INS-IGF2 transcript and the INS3 transcript expressed in case 59, we found them to have the A allele of the SNP rs689 (located at bp 216 in the INS3 transcript) (Fig. 5C and Fig 6A). The sequence containing SNP rs689 can only be found in exon 1 of INS3, not in exons of INS1, INS2 and INS4 (Fig. 1).

\section{Discussion}

Fusion genes are known to be produced by chromosomal rearrangements including small deletions, as exemplified by TMPRSS2-ERG in prostate cancer where two genes mapping to chromosome 21 are fused (24). Recently, many chimeric RNAs where adjacent genes are fused have been identified $(25,26)$. These fusion transcripts have been described by the terms 'gene read-through' and 'co-transcription and intergenic splicing' (27). Such read-through transcripts have been detected in different malignancies like prostate cancer (showing a SLC45A3-ELK4 transcript) $(25,26)$ and melanoma (with a $C D K 2-R A B 5 B$ transcript) (28). In addition to giving rise to new chimeric proteins or entirely novel proteins, read-through transcripts have been suggested to play a regulatory role by altering the expression of the parent genes (27).

Here we demonstrated, for the first time, expression of an INS-IGF2 read-through transcript in insulinomas. In some instances, the chimeric transcripts found by computation analysis of transcriptome sequencing data are false positives due to algorithm artefacts; these 'fusions' are not detectable by PCR-based methods. We could clearly detect the 
A

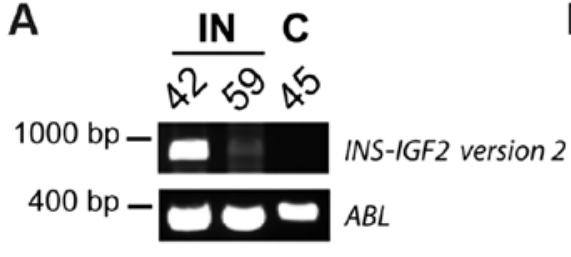

C

SEQUENCE

INS-IGF2v2

INS-IGF2v1

IGF2
B

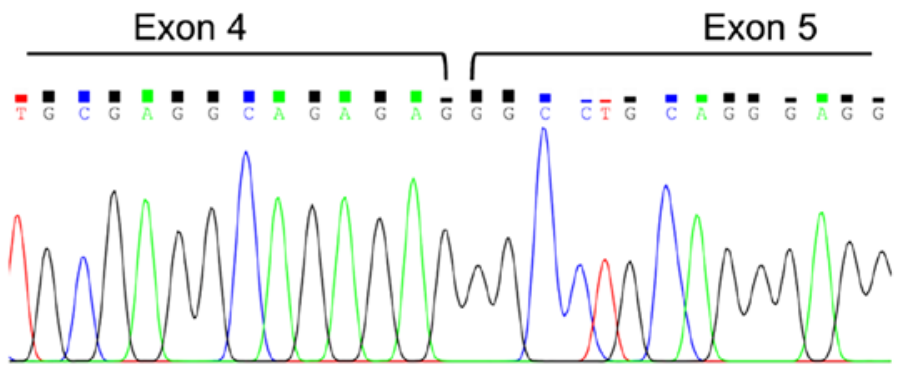

Figure 4. Detection of transcript version 2 of $I N S-I G F 2$ in insulinomas. (A) $I N S$ - IGF2 version 2 was amplified from $2 \mu 1 \mathrm{cDNA}$ with a concentration equivalent to $50 \mathrm{ng} / \mu 1$ RNA using primers 4 and 7 for $I N S$-IGF2 version 2 and primers 3 and 5 for alternatively spliced $I N S$-IGF2. ABL was amplified using primers 9 and 10 and used as an internal control for the PCR reaction. (B) Part of the sequence of the INS-IGF2 version 2 transcript amplified by RT-PCR in (A). Indicated is the exon-exon junction of exon 4 and 5 in $I N S$-IGF2 version 2. (C) Sequence alignment of the INS-IGF2 version 2 sequence amplified in (A). Exon 5 of $I N S-I G F 2$ version 2 is not present in INS-IGF2 version 1.

A

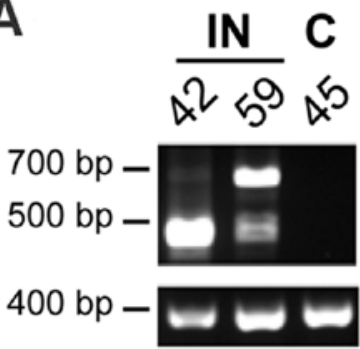

B

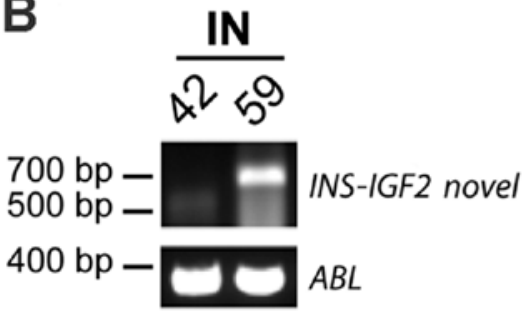

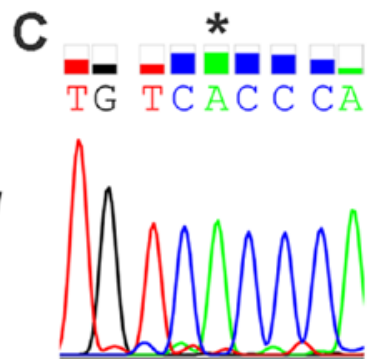

Figure 5. Detection of alternatively spliced INS-IGF2 in insulinomas. (A) Alternatively spliced INS was amplified from $0.25 \mu 1$ cDNA with a concentration equivalent to $15 \mathrm{ng} / \mu 1$ DNase-treated RNA using primers 3 and 2 for $I N S$. (B) INS-IGF2 were amplified from $1 \mu 1 \mathrm{cDNA}$ with a concentration equivalent to $50 \mathrm{ng} / \mu 1$ RNA using primers 3 and 5 for alternatively spliced $I N S-I G F 2$. Due to the limited amount of material, the control sample (case 45) was not included for alternatively spliced INS-IGF2. ABL was amplified using primers 9 and 10 and used as internal control for the PCR reaction. (C) Sequence of the A allele of the SNP rs689 in the novel splice variant of INS-IGF2. The SNP is marked by an asterisk. The sequence containing SNP rs689 is only found in exon 1 of INS3, not in exons of INS1, INS2 and INS4.

INS-IGF2 fusion transcript, identified as a fusion partner upon RNA-sequencing, by PCR-based methods, indicating that this is indeed a true fusion transcript.

The INS and IGF2 genes are located sequentially in a large imprinted domain on chromosome subband 11p15.5 (Fig. 1). Two INS-IGF2 read-through transcripts have been described that are splice variants of INS and exons of the IGF2-gene (22). Transcript 1 (long form, accession no. NR_003512.3) contains exons 1 and 2 of INSI and the 3 ' part of exon 1 of $I G F 2$ version 2 starting at bp 259, followed by exons 2-5 of IGF2 version 2 . Transcript 2 (short form, accession no. NM_001042376.2) contains exons 1 and 2 of INSI and the 3' part of exon 1 of $I G F 2$ version 2 starting at bp 259, followed by exon 2 of $I G F 2$ version 2 and an additional exon following exon 2 in the $I G F 2$ locus that is not present in the IGF2 transcripts (Fig. 1) (22).

Transcript 2 of INS-IGF2 encodes a protein which shares the N-terminus with the INS protein (signal peptide, B-chain, and eight amino acids of the $\mathrm{C}$-peptide) and has a C-terminus containing the coding sequences of the two proximal ORFs of the IGF2 gene. The IGF2-part of the INS-IGF2 protein stems from noncoding exons in the $I G F 2$ gene that give rise to a novel 138-amino acid C-terminal region unrelated to
prepro-IGF2 (22). Recently, however, Wernersson et al (29) found that although transcript 2 of INS-IGF2 can be translated (22), expression of the INS-IGF2 protein in human $\beta$-cells was below the detection level in proteomic analysis. Transcript variant 1 of INS-IGF2 is bisistronic, containing ORFs both for INS-IGF2 (same sequence as in transcript variant 2) and $I G F 2$ (transcript variant 2) (22). INS-IGF2 transcript variant 1 is a candidate for NMD as the primary ORF (INS-IGF2) has the stop codon more than 50 nucleotides from the terminal splice site. Therefore, it remains uncertain whether or not the two ORFs in transcript variant 1 are translated. Notably, our data showed that transcript variant 2 of $I N S-I G F 2$ is expressed in the insulinomas (Fig. 4), although conclusions regarding protein expression can not be made, this indicates that the INS-IGF2 chimeric protein may be expressed. Although the precise function of the INS-IGF2 protein remains unidentified, Kanatsuna et al $(30,31)$ recently found INS-IGF2 to be recognized by autoantibodies associated with diabetes mellitus type 1 (DM1). In DM1, pancreatic $\beta$-cells are destroyed due to the recognition of pancreatic islets autoantigens, like insulin, by autoreactive $\mathrm{T}$ lymphocytes leading to a T-lymphocyte-mediated immune response $(32,33)$. The 


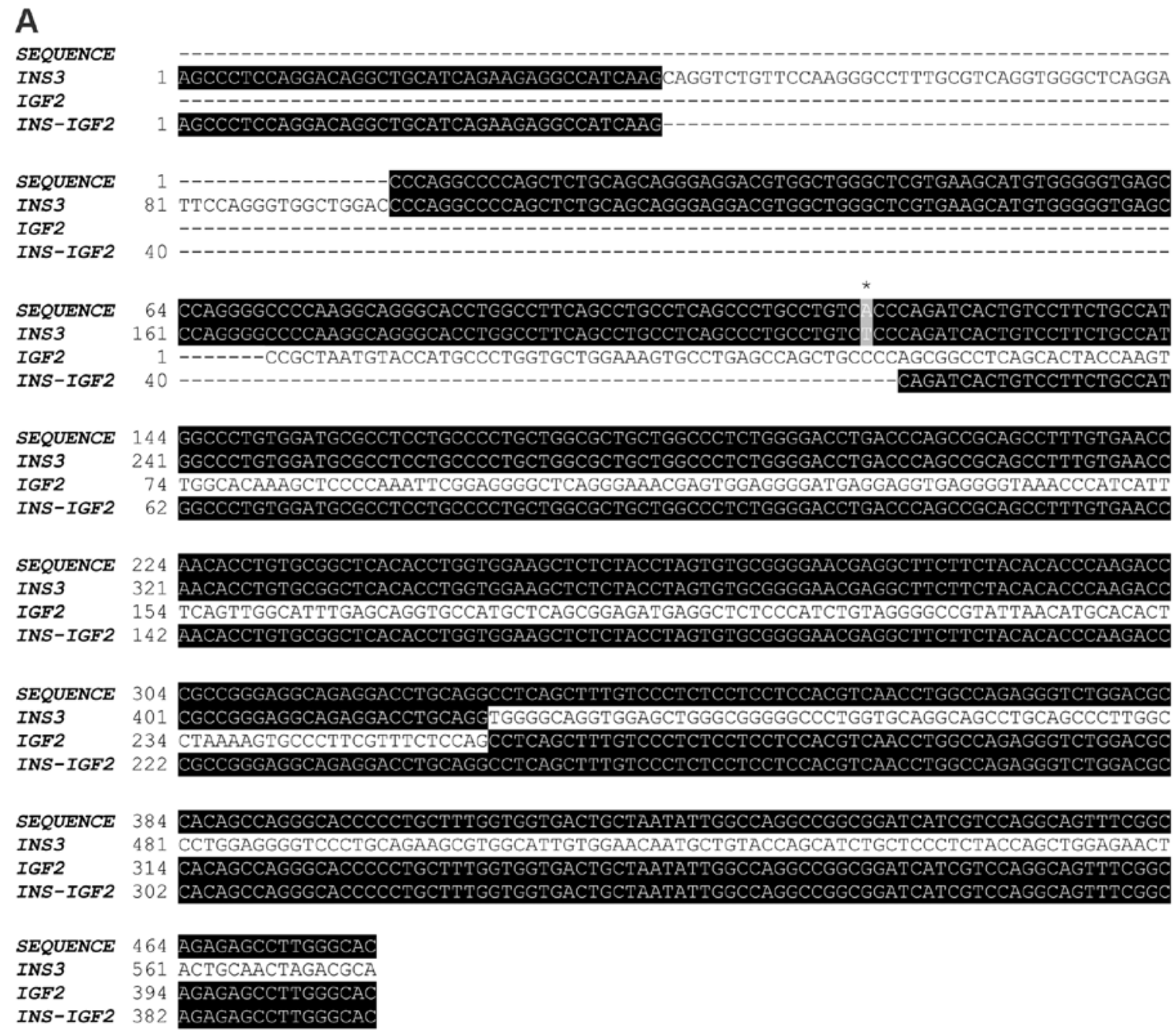

B

Transcript Version Accesion no

Schematic diagram

INS-IGF2 $\begin{cases}1 & \text { NR_003512.3 } \\ 2 & \text { NM_001042376.2 } \\ \text { Novel } & \end{cases}$

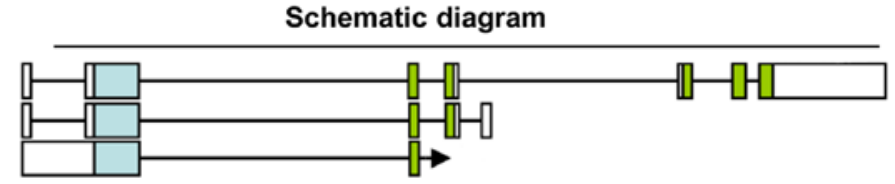

Figure 6. Sequence alignment of the novel splice variant of INS-IGF2. (A) Sequence of the novel splice variant of INS-IGF2 identified in Fig. 5B aligned to $I N S-I G F 2$, INS3 and IGF2. The SNP rs689 is marked by an asterisk. (B) Schematic diagram of the human INS-IGF2 transcripts, including the novel splice variant of INS-IGF2. Coding exons are colored.

appearance of several autoantibodies towards these pancreatic islets autoantigens is a sign of autoimmunity (32). The authors therefore suggested that INS-IGF2 may enhance the ability of insulin to trigger the development of DM1 $(30,31)$.

Normal INS-IGF2 expression is restricted to the limbs, eyes, and pancreas $(22,30)$. In the limbs and eyes, INS-IGF2 expression is maternally imprinted. However, in the pancreas the expression of INS-IGF2 is biallelic (22). Specific detection of the short transcript of INS-IGF2 demonstrated restricted expression in the human pancreas and eyes only (22). As the $I N S-I G F 2$ read-through expression is controlled by the INS transcriptional start site, one would expect the INS and $I N S$-IGF2 transcripts to show the same pattern of expression in insulinomas. We found INS expression to be higher in insulinomas compared to the control sample (Fig. 3). The same pattern of expression could be seen for INS-IGF2 although the relative expression of INS was $2 \times 10^{3}-4 \times 10^{4}$ times higher than that of INS-IGF2 in insulinomas. Notably, in a recent reanalysis of deep RNA sequencing data originally published by Nica et al (34), Wernersson et al (29) found the INS-IGF2 transcript in $\beta$-cells to have a $>20,000$ fold lower expression than the INS transcript (29), which is in line with our findings in insulinomas. Additionally, INS-IGF2 expression was below the detection level in our normal pancreatic tissue control (Fig. 3), in line with Wernersson et al (29) who found INS-IGF 2 expression to be barely detectable in normal pancreatic tissue. Kim et al (35) states that read-through transcripts are expressed at extremely low levels in human tissues with read-through transcripts showing a tumor-biased expression. Increased expression-level of read-through transcripts and increased number of read-through transcripts expressed seems therefore to be a cancer-specific event (35). 
Increased INS protein production in insulinomas can in part be explained by increased expression of the INS2 splice variant of INS where a 26 bp of intron 1 in INS1 is retained, changing the 5'-untranslated region (23). This splice variant has increased translation efficiency and the ratio of this splice variant to normal INS is increased more than 50-fold in insulinomas (23). We found INS2 and/or INS4 to be highly expressed in insulinomas but we were not able to detect an INS-IGF2 transcript with the retained 26 bp of intron 1 in INS1 (exons 1 and 2 of INS2 fused to exon 1 of IGF2), indicating that the splicing mechanisms regulating a switch from INS1 to INS2 expression does not affect the INS-IGF2 transcript. Notably, however, in one case (case 59) we found transcripts with the A allele of the SNP rs689 which has been demonstrated to alter the efficiency of intron 1 splicing in INS1, resulting in the expression of longer isoforms (36). In case 59, we found the expression of the longest INS transcript, $I N S 3$, and, of note, a novel INS-IGF2 transcript containing exon 1 of INS3 fused to exon 1 of IGF2 (Figs. 5 and 6). Both transcripts may be explained by the effect of the A allele of the SNP rs689 on splicing of intron 1 in INS1, indicating that the splicing mechanism affected by SNP rs689 similarly affects $I N S$ and $I N S-I G F 2$. The A allele of SNP rs689 is associated with increased methylation of neighboring $\mathrm{CpGs}$, especially CpG-180 whose hypermethylation is associated with DM1 (37). Furthermore, the AA genotype of the SNP rs689 is associated with DM1-risk and positivity for insulin autoantibodies (38). Based on our results we cannot determine whether case 59 has an AT or AA genotype of the SNP rs689.

Dejeux et al (39) demonstrated a correlation between differentially methylated region 2 (DMR2) hypermethylation and overexpression of IGF2 both at the RNA and the protein level. Gain of methylation in the DMR2 of the IGF2 gene distinguishes insulinomas from other PNETs (39). In correlation with the findings by Dejeux et al (39) we found $I G F 2$-expression to be increased in the two insulinomas. Interestingly, IGF2 is a regulator of cyclin D1, which has been detected in $36 \%$ of insulinomas (40), by activation of Akt upon binding to its receptor IGF-R type 2 .

Based on the present results, we cannot but speculate on the mechanism behind the expression of the INS-IGF2 read-through, whether it is due to genomic rearrangement or trans-splicing. A combination of the two mechanisms is also possible. The identification of the increased expression of the INS-IGF2 read-through transcript in tumor tissue but not in the normal pancreatic tissue used as a control, suggests that this may be a cancer-specific event. Although further studies are required, our findings may have clinical applications as INS-IGF2 could potentially be a biomarker candidate for insulinoma patients.

\section{Acknowledgements}

This study was supported by grants from the Norwegian Cancer Society; the Research Council of Norway through its Centres of Excellence funding scheme, project no. 179571; Carcinor, the Norwegian patient advocacy association for neuroendocrine cancer; the Norwegian Society of Gastroenterology, and the Henrik Homan Foundation. The authors thank Lisbeth Haugom and Dr Jim Thorsen for expert technical help.

\section{References}

1. Service FJ, McMahon MM, O'Brien PC and Ballard DJ: Functioning insulinoma-incidence, recurrence, and long-term survival of patients: A 60-year study. Mayo Clin Proc 66: 711-719, 1991.

2. Hirshberg B, Livi A, Bartlett DL, Libutti SK, Alexander HR, Doppman JL, Skarulis MC and Gorden P: Forty-eight-hour fast: The diagnostic test for insulinoma. J Clin Endocrinol Metab 85: 3222-3226, 2000.

3. Sarmiento JM, Que FG, Grant CS, Thompson GB, Farnell MB and Nagorney DM: Concurrent resections of pancreatic islet cell cancers with synchronous hepatic metastases: Outcomes of an aggressive approach. Surgery 132: 976-982; discussion 982-983, 2002.

4. Danforth DN Jr, Gorden P and Brennan MF: Metastatic insulin-secreting carcinoma of the pancreas: Clinical course and the role of surgery. Surgery 96: 1027-1037, 1984.

5. Hirshberg B, Cochran C, Skarulis MC, Libutti SK, Alexander HR, Wood BJ, Chang R, Kleiner DE and Gorden P: Malignant insulinoma: Spectrum of unusual clinical features. Cancer 104: 264-272, 2005 .

6. La Rosa S, Pariani D, Calandra C, Marando A, Sessa F, Cortese F and Capella C: Ectopic duodenal insulinoma: A very rare and challenging tumor type. Description of a case and review of the literature. Endocr Pathol 24: 213-219, 2013.

7. Stefanini P, Carboni M, Patrassi N and Basoli A: Beta-islet cell tumors of the pancreas: Results of a study on 1,067 cases. Surgery 75: 597-609, 1974.

8. Haugvik SP, Marangos IP, Røsok BI, Pomianowska E, Gladhaug IP, Mathisen O and Edwin B: Long-term outcome of laparoscopic surgery for pancreatic neuroendocrine tumors. World J Surg 37: 582-590, 2013.

9. Jonkers YM, Ramaekers FC and Speel EJ: Molecular alterations during insulinoma tumorigenesis. Biochim Biophys Acta 1775: 313-332, 2007.

10. Capurso G, Festa S, Valente R, Piciucchi M, Panzuto F, Jensen RT and Delle Fave G: Molecular pathology and genetics of pancreatic endocrine tumours. J Mol Endocrinol 49: R37-R50, 2012.

11. Speel EJ, Scheidweiler AF, Zhao J, Matter C, Saremaslani P, Roth J, Heitz PU and Komminoth P: Genetic evidence for early divergence of small functioning and nonfunctioning endocrine pancreatic tumors: Gain of $9 \mathrm{Q} 34$ is an early event in insulinomas. Cancer Res 61: 5186-5192, 2001.

12. Jonkers YM, Claessen SM, Feuth T, van Kessel AG, Ramaekers FC, Veltman JA and Speel EJ: Novel candidate tumour suppressor gene loci on chromosomes 11q23-24 and 22 q13 involved in human insulinoma tumourigenesis. J Pathol 210: 450-458, 2006.

13. Jonkers YM, Claessen SM, Perren A, Schmid S, Komminoth P, Verhofstad AA, Hofland LJ, de Krijger RR, Slootweg PJ, Ramaekers FC, et al: Chromosomal instability predicts metastatic disease in patients with insulinomas. Endocr Relat Cancer 12: 435-447, 2005.

14. Speel EJ, Richter J, Moch H, Egenter C, Saremaslani P, Rütimann K, Zhao J, Barghorn A, Roth J, Heitz PU, et al: Genetic differences in endocrine pancreatic tumor subtypes detected by comparative genomic hybridization. Am J Pathol 155: 1787-1794, 1999.

15. Chung DC, Brown SB, Graeme-Cook F, Tillotson LG, Warshaw AL, Jensen RT and Arnold A: Localization of putative tumor suppressor loci by genome-wide allelotyping in human pancreatic endocrine tumors. Cancer Res 58: 3706-3711, 1998.

16. Stumpf E, Aalto Y, Höög A, Kjellman M, Otonkoski T, Knuutila $S$ and Andersson LC: Chromosomal alterations in human pancreatic endocrine tumors. Genes Chromosomes Cancer 29: 83-87, 2000

17. Zhao J, Moch H, Scheidweiler AF, Baer A, Schäffer AA, Speel EJ, Roth J, Heitz PU and Komminoth P: Genomic imbalances in the progression of endocrine pancreatic tumors. Genes Chromosomes Cancer 32: 364-372, 2001.

18. Capdeville R, Buchdunger E, Zimmermann J and Matter A: Glivec (STI571, imatinib), a rationally developed, targeted anticancer drug. Nat Rev Drug Discov 1: 493-502, 2002.

19. Haugvik SP, Gorunova L, Haugom L, Eibak AM, Gladhaug IP, Heim S and Micci F: Loss of 11p11 is a frequent and early event in sporadic nonfunctioning pancreatic neuroendocrine neoplasms. Oncol Rep 32: 906-912, 2014. 
20. Ge H, Liu K, Juan T, Fang F, Newman M and Hoeck W: FusionMap: Detecting fusion genes from next-generation sequencing data at base-pair resolution. Bioinformatics 27: 1922-1928, 2011

21. Livak KJ and Schmittgen TD: Analysis of relative gene expression data using real-time quantitative PCR and the $2^{-\Delta \Delta C T}$ method. Methods 25: 402-408, 2001.

22. Monk D, Sanches R, Arnaud P, Apostolidou S, Hills FA, Abu-Amero S, Murrell A, Friess H, Reik W, Stanier P, et al: Imprinting of $I G F 2 \mathrm{P} 0$ transcript and novel alternatively spliced $I N S-I G F 2$ isoforms show differences between mouse and human. Hum Mol Genet 15: 1259-1269, 2006.

23. Minn AH, Kayton M, Lorang D, Hoffmann SC, Harlan DM, Libutti SK and Shalev A: Insulinomas and expression of an insulin splice variant. Lancet 363: 363-367, 2004.

24. Yoshimoto M, Joshua AM, Chilton-Macneill S, Bayani J, Selvarajah S, Evans AJ, Zielenska M and Squire JA: Three-color FISH analysis of TMPRSS2/ERG fusions in prostate cancer indicates that genomic microdeletion of chromosome 21 is associated with rearrangement. Neoplasia 8: 465-469, 2006.

25. Rickman DS, Pflueger D, Moss B, VanDoren VE, Chen CX, de la Taille A, Kuefer R, Tewari AK, Setlur SR, Demichelis F, et al: SLC45A3-ELK4 is a novel and frequent erythroblast transformation-specific fusion transcript in prostate cancer. Cancer Res 69: 2734-2738, 2009.

26. Zhang Y, Gong M, Yuan H, Park HG, Frierson HF and Li H: Chimeric transcript generated by cis-splicing of adjacent genes regulates prostate cancer cell proliferation. Cancer Discov 2: 598-607, 2012

27. Prakash T, Sharma VK, Adati N, Ozawa R, Kumar N, Nishida Y, Fujikake T, Takeda T and Taylor TD: Expression of conjoined genes: Another mechanism for gene regulation in eukaryotes. PLoS One 5: e13284, 2010.

28. Berger MF, Levin JZ, Vijayendran K, Sivachenko A, Adiconis X, Maguire J, Johnson LA, Robinson J, Verhaak RG, Sougnez C, et al: Integrative analysis of the melanoma transcriptome. Genome Res 20: 413-427, 2010.

29. Wernersson R, Frogne T, Rescan C, Hansson L, Bruun C, Grønborg M, Jensen JN and Madsen OD: Analysis artefacts of the INS-IGF2 fusion transcript. BMC Mol Biol 16: 13, 2015.

30. Kanatsuna N, Taneera J, Vaziri-Sani F, Wierup N, Larsson HE, Delli A, Skärstrand H, Balhuizen A, Bennet H, Steiner DF, et al: Autoimmunity against INS-IGF2 protein expressed in human pancreatic islets. J Biol Chem 288: 29013-29023, 2013.
31. Kanatsuna N, Delli A, Andersson C, Nilsson AL, Vaziri-Sani F, Larsson K, Carlsson A, Cedervall E, Jönsson B, Neiderud J, et al: Doubly reactive INS-IGF2 autoantibodies in children with newly diagnosed autoimmune (type 1) diabetes. Scand J Immunol 82: 361-369, 2015.

32. Skyler JS: Immune intervention for type 1 diabetes mellitus. Int J Clin Pract Suppl 65: 61-70, 2011.

33. Moser A, Hsu HT and van Endert P: Beta cell antigens in type diabetes: Triggers in pathogenesis and therapeutic targets. F1000 Biol Rep 2: 75, 2010.

34. Nica AC, Ongen H, Irminger JC, Bosco D, Berney $T$, Antonarakis SE, Halban PA and Dermitzakis ET: Cell-type, allelic, and genetic signatures in the human pancreatic beta cell transcriptome. Genome Res 23: 1554-1562, 2013.

35. Kim RN, Kim A, Choi SH, Kim DS, Nam SH, Kim DW, Kim DW, Kang A, Kim MY, Park KH, et al: Novel mechanism of conjoined gene formation in the human genome. Funct Integr Genomics 12: 45-61, 2012.

36. Kralovicova J and Vorechovsky I: Allele-specific recognition of the 3' splice site of INS intron 1. Hum Genet 128: 383-400, 2010.

37. Fradin D, Le Fur S, Mille C, Naoui N, Groves C, Zelenika D, McCarthy MI, Lathrop M and Bougnères P: Association of the $\mathrm{CpG}$ methylation pattern of the proximal insulin gene promoter with type 1 diabetes. PLoS One 7: e36278, 2012.

38. Lempainen J, Härkönen T, Laine A, Knip M and Ilonen J; Finnish Pediatric Diabetes Register: Associations of polymorphisms in non-HLA loci with autoantibodies at the diagnosis of type 1 diabetes: INS and IKZF4 associate with insulin autoantibodies. Pediatr Diabetes 14: 490-496, 2013.

39. Dejeux E, Olaso R, Dousset B, Audebourg A, Gut IG, Terris B and Tost J: Hypermethylation of the IGF2 differentially methylated region 2 is a specific event in insulinomas leading to loss-of-imprinting and overexpression. Endocr Relat Cancer 16: 939-952, 2009.

40. Chung DC, Brown SB, Graeme-Cook F, Seto M, Warshaw AL, Jensen RT and Arnold A: Overexpression of cyclin D1 occurs frequently in human pancreatic endocrine tumors. J Clin Endocrinol Metab 85: 4373-4378, 2000. 